\title{
Grenzen in Bewegung 1
}

\section{Moving borders}

\author{
Thomas Nail, Denver
}

Zusammenfassung: Dieser Beitrag schlägt einen neuen, bewegungsorientierten grenztheoretischen Ansatz vor. Gegen politikphilosophische Konzeptionen, die Grenzen als statisch und unpassierbar fassen, wird unter Verweis auf historische und empirische Studien gezeigt, dass und inwiefern Grenzen dehnbar, fluktuierend und beweglich sind. Auf dieser Grundlage wird im folgenden Abschnitt argumentiert, dass sich dynamische Prozesse der Grenzziehung am angemessensten in Sinne von Zirkulation begreifen lassen: Anstatt Mobilität zu verhindern und stabile Formen von Ein- bzw. Ausschluss zu etablieren, stellen Grenzen Regime sozialer Zirkulation dar, welche Menschen, deren Arbeitskraft und Steuern im Interesse der Schaffung gesellschaftlicher Ordnung und wirtschaftlichen Mehrwerts regulieren. Unter Rückgriff auf Marx' Konzept der ursprünglichen Akkumulation präsentiere ich anschließend eine Neubeschreibung von Grenzen als beweglichen Instrumenten ökonomischer und sozialer Aneignung - ich bringe dies auf den Begriff „Ausdehnung durch Ausschließung“. Im abschließenden Abschnitt untersuche ich, welche Gestalt „Ausdehnung durch (territoriale, politische, rechtliche und wirtschaftliche) Ausschließung“ unter den Bedingungen von Klimawandel annimmt und inwiefern dabei Bevölkerungsgruppen produziert werden, die in höchstem Maße verletzlich und ausbeutbar sind.

Schlagwörter: Mobilität - Flucht - Kinopolitik - (Grenze und) Zirkulation - (Grenze und) Akkumulation

1 Der Originalbeitrag „Moving borders“ ist im von Anthony Cooper und Søren Tinning herausgegebenen Band Debating and Defining Borders: Philosophical and Theoretical Perspectives (London: Routledge, 2019), 195-205, erschienen. Übersetzung von Florian Grosser. 
Abstract: This article proposes a new, movement-oriented approach to theorizing borders. In contradistinction to politico-philosophical conceptions of borders as fixed and impassible, it points to historical and empirical evidence to show that borders are malleable, fluctuating, and constantly in motion. On this basis, it is further argued that dynamic processes of bordering are best understood in terms of circulation: Rather than preventing movement and establishing stable forms of inclusion and exclusion, borders are regimes of social circulation which regulate humans, labor, and taxes in the interest of ordering society and extracting an economic surplus. Drawing on Marx's concept of primitive accumulation, I subsequently redescribe borders as mobile tools of economic as well as social accumulation - of what I call "expansion by expulsion". In the final section, I examine how "expansion by (territorial, political, juridical, and economic) expulsion" operates under conditions of climate change so as to produce highly vulnerable and exploitable populations.

Keywords: Mobility - refugees - kinopolitics - (border and) circulation - (border and) accumulation

Dieser Text zu Grenzen in Bewegung² legt eine neue, prozess- bzw. bewegungsorientierte oder „kinopolitische“ Methodologie vor, um Grenzen zu studieren. Es sind zwei verbreitete Annahmen darüber, wie Grenzen funktionieren, gegen die ich hier argumentieren möchte: Die Annahme, dass Grenzen statisch sind, sowie die Annahme, dass sie Menschen abhalten und ihnen Eintritt verwehren.

Mein Argument wird in Form von drei eng miteinander verzahnten Thesen entwickelt: (1) Grenzen sind in Bewegung; (2) deren zentrale Funktion besteht nicht darin, Bewegung aufzuhalten, sondern diese zirkulieren zu lassen; und (3) Grenzen sind Werkzeuge ursprünglicher Akkumulation. Auf die Erörterung dieser drei Thesen folgt zu Illustrationszwecken ein konkretes Fallbeispiel. Wie ich an anderer Stelle ausführlich aufgezeigt habe, haben diese Thesen erhebliche Auswirkungen darauf, wie Grenzen heute neu zu theorisieren sind (siehe Nail 2016).

Sich mit Grenzen auseinanderzusetzen ist gegenwärtig wichtiger denn je. Zu Beginn des 21. Jahrhunderts wurden mehr Migrant*innen als jemals

2 Der Originaltitel meines Aufsatzes ist Peter Nyers wunderbarem Essay „Moving Borders: The Politics of Dirt“ entliehen, der in Radical Philosophy 174 (Juli/August 2012) erschienen ist. Ich bin Peter dankbar für seine Unterstützung meiner Forschung und allgemein für seine Beiträge auf dem Feld der Border Studies. 
zuvor registriert (siehe IOM 2010; WHO 2015);3 deren Zahl liegt jenseits von einer Milliarde (siehe UNDP 2009). ${ }^{4}$ Seit Beginn dieses Jahrhunderts hat Migration um etwa 50 Prozent zugenommen, dabei sind allein in den Jahren 2013 bis 2017 über 56.0oo Migrant*innen zu Tode gekommen bzw. verschwunden (siehe Hinnant und Janssen 2018). Aufgrund von ökologischer, ökonomischer und politischer Instabilität sind immer mehr Menschen zu Migration gezwungen. Insbesondere bedingt durch Klimawandel könnte sich der Umfang internationaler Migration über die nächsten 40 Jahre sogar verdoppeln (siehe IOM 2009). ${ }^{5}$ Darüber hinaus wächst auch der Prozentsatz an Migrant*innen, die keinen Rechtsstatus besitzen und die undokumentiert sind - woraus sich ernste Herausforderungen für liberale Demokratien ergeben, die auf dem Prinzip universeller Gleichheit basieren. ${ }^{6}$

Um die in diesem Maße zunehmende globale Mobilität zu steuern und zu kontrollieren, wird die Welt stärker als je zuvor von Grenzziehungen geprägt. Allein in den vergangenen 20 Jahren sind in Reaktion auf die Terroranschläge in den USA am 11. September 2001 oder, in jüngerer Vergangenheit, auf den Krieg in Syrien weltweit Hunderte von zusätzlichen Grenzen entstanden: Meilen von Stacheldrahtzäunen und Betonmauern, dazu zahlreiche offshore detention centers, Datenbanken für biometrische Reisepässe sowie Kontrollpunkte in Schulen, an Flughäfen und entlang verschiedenster Verkehrswege. In alledem manifestiert sich, was seit jeher den strategischen Kern des globalen Kapitalismus und Kolonialismus bildet - nämlich eine Aneignung der Reichtümer der Welt und ein Aussperren derer, die in Armut

3 Der Internationalen Organisation für Migration und der Weltgesundheitsorganisation zufolge liegt deren Gesamtzahl bei etwa einer Milliarde oder, in Prozenten gerechnet, bei etwa 14 Prozent der Weltbevölkerung.

Für das Jahr 2010 verzeichnet der „Bericht über die menschliche Entwicklung“ der Vereinten Nationen 215 Millionen internationaler sowie 740 Millionen interner Migrant*innen. Nach Schätzungen der Vereinten Nationen aus dem Jahr 2019 liegt die Zahl internationaler Migrant*innen bei 272 Millionen, von denen über 70 Millionen aufgrund von Konflikten auf der Flucht sind.

Vorhersagen für die Zukunft variieren zwischen 25 Millionen und 1 Milliarde Klimaflüchtlingen, die sich bis zum Jahr 2050 vorläufig oder dauerhaft innerhalb ihrer Herkunftsländer oder über Grenzen hinweg bewegen werden. Die häufigste Schätzung liegt bei 200 Millionen, was in etwa der gegenwärtigen Gesamtzahl internationaler Migrant*innen weltweit entspricht.

$6 \quad \mathrm{Zu}$ den theoretischen Implikationen dieses Phänomens für den Liberalismus siehe Cole 2000. 
leben. Bruno Latour bemerkt dazu: „Europe has invaded all peoples; all peoples are coming to Europe in their turn." (Latour 2018, 103).

Das rezente (Wieder-)Aufkommen von rechtsgerichtetem Nationalismus und von Fremdenfeindlichkeit in westlichen Ländern ist als Reaktion ebendarauf, d.h. auf eine so genannte „migrantische Invasion“, zu verstehen. Grenzen sind die neuen Waffen dieser Länder, um ihren Kampf gegen den Rest der Welt fortzusetzen. Dies ist der Kontext, aus dem sich die Wichtigkeit ergibt, Grenzen heute neu zu denken.

\section{These 1: Die Grenze ist in Bewegung}

Auf den ersten Blick handelt es sich hierbei um eine äußerst kontraintuitive These. Worauf ich damit hinauswill, ist, dass das Problem nicht darin besteht, dass die Grenze im Übermaß fixiert und unpassierbar ist, sondern dass gerade das Gegenteil der Fall ist! Da die Grenze eminent dehnbar und fluktuierend ist - sich also kontinuierlich zwischen den zwei Seiten bewegt, welche sie voneinander trennt -, verändert sie letztlich die Topologie beider Seiten und damit auch der Figuren, die auf diesen Seiten bzw. durch diese definiert werden. Grenzen sind nicht statisch, in Abhängigkeit von einer Unzahl von Variablen werden sie immer wieder neu geschaffen. Insofern sollte die Grenze nicht einfach deswegen unter Vorzeichen von Bewegung analysiert werden, weil sich Personen und Objekte über sie bewegen oder weil sie „durchlässig“ ist. Die Grenze ist nicht lediglich eine stationäre Membran oder ein Raum, durch den Menschen- und Güterströme fließen. Im Unterschied zu der umfangreichen Literatur, die sich mit der grenzübergreifenden Bewegung von Personen und Dingen auseinandersetzt, gibt es leider vergleichsweise wenige Analysen zur Bewegung der Grenze selbst. Sogar viele der so genannten Theoretiker*innen des flow, der Fluidität und Mobilität, beschreiben die Grenze nach wie vor vornehmlich in Begriffen der Ausdehnung und Räumlichkeit - so etwa als von „global flows of people“ gestaltete Grenzlandschaften (siehe Latour 2018) oder als ,the material form of support for flows" (Castells 1996, 376), deren mobiler oder fluider Charakter ausschließlich „metaphorisch“ verstanden wird.7

Die Bewegung der Grenze ist jedoch keine Metapher, ist die Grenze doch buchstäblich und tatsächlich in Bewegung. Erstens bewegt sich die Grenze selbst. Dies wird am Fall der Geomorphologie besonders deutlich,

$7 \quad$ Für Beispiele eines metaphorischen Gebrauchs der Konzepte von Mobilität und Fluidität siehe Urry 2000; Bauman 2013. 
d.h. an der Eigenbewegtheit von Flüssen, an sich verlagernden Sandformationen, Strömungen an Küstenlinien und so weiter. Die Grenze bewegt sich auch in weniger offensichtlicher Weise selbst, so z. B. durch Erosion, Verfall und Dekomposition, die Grenzbefestigungen ebenso konstant betreffen wie jedes andere physische Objekt auf Erden. Das beinhaltet das Zerbröckeln des Mörtels, der Mauern zusammenhält; Regen und Fluten, die hölzerne Zäune verfallen lassen; Feuer, die Gebäude und Wachtürme zerstören; Rost, der Löcher in Zäune und Tore frisst; Auswaschung und Abtragung, die Erdreich entfernen und verschieben; oder Stürme auf dem Mittelmeer. Dass die Grenze in ihrer Materialität Bewegungen der Selbstzersetzung unterliegt, hat Konsequenzen für Migrant*innen, die beispielsweise Schwachstellen in Grenzbefestigungen nutzen, um diese zu überqueren. Zum Teil werden derartige Schwachstellen von staatlichen Autoritäten bewusst nicht behoben, um Migrant*innen so in lebensbedrohliche Situationen zu bringen - zu denken wäre hier etwa an den Devil's Highway im Süden von Arizona oder an gefährliche Seerouten.

Zweitens wird die Grenze auch von anderen bewegt. Dies wird im Fall von territorialen Konflikten besonders offenkundig, in denen zwei oder mehr Parteien über die Aufteilung von Gebieten verhandeln oder kämpfen; von politischen und militärischen Konflikten, in denen es um die Kontrolle über Menschen, Land und Ressourcen geht; von juridischen Teilungen von Rechtsbereichen oder polizeilichen Gebietskörperschaften; sowie von wirtschaftlichen Reformen, die Handelsbarrieren, Tarife, Arbeitsbeschränkungen und Produktionsgebiete unmittelbar verändern. Grenzen, die große zonenartige Gebiete umfassen, können als Stätten fortgesetzter Aushandlung und Bewegung bestehen, so etwa die Siedlungen im Westjordanland. Der Status von Migrant*innen als feindlichen Kämpfer*innen oder Siedler*innen schwankt und wandelt sich dabei zusammen mit den Wandlungen der Grenze.

Doch auch in diesem Fall gibt es weniger augenfällige Arten und Weisen, in welchen die Grenze bewegt wird, so etwa durch stetige Prozesse des Managements, die erforderlich sind, um sie aufrechtzuerhalten. Ohne regelmäßige Interventionen und Neuproduktionen (oder auch rechtliche und wirtschaftliche Eingriffe) verfallen Grenzen, werden vergessen, geschwächt oder schlicht von anderen eingenommen. Grenzen sind weder feststehend noch einfach gegeben, sie werden stattdessen wieder und wieder geschaffen, kinetisch wie materiell. Zu diesem Sachverhalt schreibt Nick Vaughan-Williams: 
None of these borders is in any sense given but (re)produced through modes of affirmation and contestation and is, above all, lived. In other words borders are not natural, neutral nor static but historically contingent, politically charged, dynamic phenomena that first and foremost involve people and their everyday lives. (Vaughan-Williams 2009, 1)

Freilich ermöglicht ebendiese Tatsache den willkürlichen Einsatz von Polizeigewalt, das Profiling von Migrant*innen oder Mikro-Ökonomien der Bestechung. Selbst in sanctuary cities in den USA kann jeder Migrant*innen bei den Einwanderungsbehörden melden, sofern der Verdacht besteht, dass diese sich irregulär dort aufhalten - tatsächlich jede* ${ }^{*}$ kann einer Grenze Geltung verschaffen, sogar Migrant*innen selbst.

Das vorherrschende geistige Bild, das viele von Grenzen als statischen Mauern haben, ist weder konzeptuell noch praktisch zutreffend. Vielmehr ähneln Grenzen Gabelungspunkten oder Motoren. Wie andere Motoren, so müssen auch Grenztechnologien in Stand gehalten und aufs Neue betankt, repariert und gestartet werden. Auch ethnische, religiöse oder nationale Grenzen besitzen ihre spezifischen Technologien, die sich beispielsweise in der Kontrolle darüber niederschlagen, wer Zutritt zu welchem Café, welcher Kirche oder welcher Schule hat. Ferner handelt es sich bei bewegten Grenzen nicht um ein ausschließlich oder vornehmlich zeitgenössisches Phänomen (siehe Johnson et al. 2011) - denn immer schon sind Grenzen in mobiler und multipler Gestalt aufgetreten, in der einen oder anderen Weise ist Management immer schon ein Bestandteil von deren Existenz gewesen. ${ }^{8}$

Angesichts dessen lässt sich die Unterscheidung zwischen natürlichen und künstlichen Grenzen nicht aufrechterhalten, die in frühen Grenztheorien eingeführt wurde. ${ }^{9}$ Das liegt keineswegs daran, dass Grenzen heute radikal anders sind als in der Vergangenheit; es liegt vielmehr daran, dass vermeintlich „natürliche“ Grenzen im Laufe der Geschichte stets erst durch

8 Dass Grenzen beweglich sind und deren Management von zentraler Bedeutung ist, stellt kein neues Phänomen dar - anders als von manchen argumentiert wird: „If the major focus of past research into borders was concerned with the way in which they were demarcated and delimited, it is the management of the border regime which is of greater importance today." (Newman 2003, 16)

9 Für eine Zusammenfassung historischer Positionen, die auf der Differenz zwischen natürlichen und künstlichen Grenzen beharren, siehe Prescott 1987; Ancel 1936. 
„künstliche“ menschliche Verbände und Gesellschaften als Grenzen gezogen, ausverhandelt und aufrechterhalten wurden. So kann ein Fluss nur als Grenze funktionieren, wenn er sich in irgendeiner Form - z. B. vermittelt durch eine Brücke, eine Steuer, eine gesellschaftlich umstrittene oder akzeptierte Trennung - sozial niederschlägt. Außerdem erfüllen so genannte künstliche Grenzen ihre Funktion immer, indem sie den „natürlichen“ Strom der Erdbewegungen oder der Bewegungen von Menschen (die selbst „natürliche“ Lebewesen sind) durchschneiden oder unterteilen. Ein drastisches Beispiel hierfür stellen Versuche der US-Regierung dar, die natürlicherweise „unsichere" Topologie der Grenze zu Mexiko außerhalb von San Diego dadurch zu verändern, dass 1,5 Millionen Kubikmeter Erde (eine Menge, die ausreicht, das gesamte Empire State Building zu füllen) von einem nahe gelegenen Berg abgetragen und zu Zwecken der Grenzsicherung verbaut werden. Dies hatte einzig den Effekt, dass die verschobene Erde innerhalb weniger Monate erodierte, freilich nicht, ohne sowohl Straßenbefestigungen als auch das Ökosystem zu beschädigen.

Ebenso wie Grenzen sich in dieser Weise bewegen und verschieben, bewegen und verschieben sich auch die migrantischen Positionen, die von ihnen abgesteckt werden. So kann es etwa passieren, dass man aufgrund von Militärmaßnahmen, die die russischen Grenzen auszudehnen suchen, in Georgien schlafen geht - und als festgenommener irregulärer Migrant aufwacht. Oder dass man auf einem Flug von Europa in die Vereinigten Staaten einschläft, um bei Ankunft gemäß der Einreiseverbote Donald Trumps als Terrorverdächtiger wieder aufzuwachen.

\section{These 2: Die Grenze ist ein Zirkulationsprozess}

Grenzen lassen sich nicht in Bezug auf Inklusion und Exklusion fassen, sie sind stattdessen erst im Sinne von Zirkulation angemessen zu begreifen. Das ergibt sich zum Teil aus der geschilderten Beweglichkeit der Grenze. Da die Grenze in einem Zwischen situiert und in Bewegung ist, stellt sie einen Prozess stetiger Verwandlung dar. Grenzen sind zu keinem Zeitpunkt einfach damit fertig, jemanden oder etwas „einzuschließen“. Dies ist nicht allein deshalb der Fall, weil Grenzen empirisch sowohl an Randgebieten der Gesellschaft als auch in deren Innerem gelegen sind, so dass sich durch sie vollzogene Auswahlprozesse des Einschlusses regelmäßig verändern; es liegt auch daran, dass auch Ausschluss nicht zusammenfällt mit stasis. Vielmehr sind auch Ausschlüsse in Bewegung und zirkulieren fortwährend. 
In der Praxis haben es Innen- wie Außengrenzen noch nie vermocht, Menschen zuverlässig innerhalb bzw. außerhalb bestimmter Bereiche zu halten. Angesichts ihres fortwährenden Scheiterns in dieser Beziehung verlieren die binären und abstrakten Kategorien von Ein- und Ausschluss somit fast jegliche Erklärungskraft. Insofern Grenzen immer schon löchrig waren, verweist deren Scheitern, umfassend ein- bzw. auszuschließen, nicht nur auf die gegenwärtig schwindende staatliche Souveränität unter postnationalen Bedingungen (siehe Brown 2010). Selbst die vermeintlich wichtigsten historischen Beispiele für die Macht von Mauern (wall power) - der Hadrianswall oder die Chinesische Mauer - waren nicht dazu gedacht, Menschen in absoluter Weise von Eintritt und Übertritt abzuhalten. Vielmehr bestand deren Funktion in der sozialen Zirkulation von Arbeitskraft und von Steuern (siehe Nail 2016, insbesondere Kapitel 3). Ebendiese Funktion erfüllt heute auch die Grenzmauer zwischen den Vereinigten Staaten und Mexiko (siehe Nail 2016, Teil III). So liegt die Erfolgsquote illegaler Grenzüberquerungen Studien zufolge bei etwa 90 Prozent; der Großteil dieses grenzübergreifenden Verkehrs steht in Verbindung mit wirtschaftlicher Regulierung. Daraus ist zu ersehen, inwiefern die wesentlichen Effekte von Grenzen nicht darin bestehen, Körper auszuschließen, sondern darin, diese gemäß bestimmter Muster in Umlauf zu bringen - so etwa, um sie zu kriminalisieren, zu töten oder um aus diesen Steuereinnahmen zu generieren. Die amerikanisch-mexikanische Grenze steht daher nicht für ein Scheitern, vielmehr erweist sie sich als erfolgreich darin, „Trichter-“ wie auch „Käfigeffekte“ zu erzielen.

Jedoch handelt es sich bei derartigen Grenzzirkulationen insofern nicht nur um einen fortgesetzten Prozess der Teilung und Trennung, als die dazu verwendeten Technologien sich auch unmittelbar auf die Elemente auswirken, die geteilt und voneinander getrennt werden. So muss, was geteilt wird, einerseits erneut in Umlauf gebracht, aufrechterhalten und sogar erweitert werden. Andererseits muss es aber auch ab- und ausgestoßen werden. Damit stehen diese Formen der Teilung nicht einfach für Blockade, sie stellen Mechanismen der Umleitung und Neuausrichtung dessen dar, was geteilt wurde. Was einmal in Umlauf gebracht worden ist, kommt auch nach erfolgter Teilung und Trennung nicht zum Stillstand - es kommt wieder und wieder, zirkuliert immer weiter. Wie David Newman zeigt, ist es also nicht die Grenzlinie, sondern der „process of bordering“ (Newman 2003, 15), welcher für gesellschaftliche Ordnung umfassende Bedeutung besitzt. Die Grenze erweist sich als eine soziale Technik, die Grenzpunkte schafft, von denen aus bzw. nach deren Regeln diejenigen, die in Zirkulation versetzt werden, 
immer wieder und zu bestimmten Bedingungen wiederkommen können (so z. B. in den Rollen der Arbeitskraft, des Kriminellen oder des Pendlers).

Wie etwa Giorgio Agamben argumentiert, trifft die Grenze ihre „Entscheidungen“ (über Trennung, Zirkulation und Verteilung) nicht logisch, sondern praktisch (siehe Agamben 1998). Zum Beispiel werden undokumentierte Migrant*innen zumeist nicht blockiert, sie werden vielmehr „kriminalisiert“ und anschließend geradezu zweckmäßig über verschiedene Bereiche der Schattenwirtschaft verteilt. In anderen Fällen lässt sich ein ökonomischer Mehrwert aus deren inhaftierten Körpern extrahieren, die in den privatwirtschaftlichen gefängnisindustriellen Komplex eingespeist werden (so erhalten private Gefängnisunternehmen aus staatlichen Zuschüssen über Jahre hinweg 200 Dollar pro Nacht für jedes belegte Bett, d. h. für jede festgehaltene Person). Sie werden nur entlassen, um auf der anderen Seite der Gefängnismauern erneut in einen Prozess zu geraten, der letztlich ein ganzes Regime sozialer Zirkulation hervorbringt, nämlich den Komplex der Abschiebungsindustrie.

Da die Grenze kein logischer, binärer oder souveräner Schnitt oder Trennstrich ist, brechen die von ihr vollzogenen Zirkulationsprozesse oftmals auch zusammen oder funktionieren nur partiell, wodurch sich Teilungen vervielfältigen oder verlagern können. Anstatt gemäß der statischen Binärlogik der Souveränität in zwei zu teilen, produziert die Grenze daher eine Vielzahl von Bifurkationen: Sie fügt zu jeder einzelnen Gabelung weitere Gabelungen hinzu und verzweigt sich so immer weiter. In Abwandlung von Carl Schmitts Formel, dass souverän ist, wer über den Ausnahmezustand entscheidet, wäre angesichts dessen zu sagen, dass es die Grenze ist, die über Teilungen entscheidet und diese in Umlauf bringt. Um das bisher Gesagte noch einmal zusammenzufassen: Grenzen sind erheblich beweglicher, als wir üblicherweise denken. Und ihre maßgebliche Funktion besteht nicht darin, Bewegung aufzuhalten, sondern zirkulieren zu lassen.

\section{These 3: Grenzen sind Werkzeuge ursprünglicher Akkumulation}

Karl Marx entwickelte das Konzept der ursprünglichen Akkumulation in Auseinandersetzung mit einer Passage aus Adam Smiths The Wealth of Nations, in der es heißt: „The accumulation of stock must, in the nature of things, be previous to the division of labour." (Smith 2003, 350) Anders gesagt, muss demnach bereits vor der Aufspaltung in Eigentümer und Arbeitskräfte eine 
Form von Akkumulation existiert haben, welche es den Machthabenden ermöglicht hat, dieser Trennung überhaupt erst Geltung zu verschaffen. Die in der Geschichte überlegenen Völker häufen demnach in natürlicher Weise Macht und Bestände an, welche sie dann zur Anwendung bringen, um die Unterordnung der ihnen Unterlegenen zu perpetuieren. Smith zufolge handelt es sich dabei schlicht um ein natürliches Phänomen: Wer an der Macht ist, hat Besitzstände immer schon angehäuft, und dies wie aus dem Nichts.

Für Marx ist diese Passage bei Smith freilich emblematisch für die Art und Weise, in der politische Ökonomen Vertreibung und Gewalt als Voraussetzungen für den Erhalt von Macht und die Vermehrung von Besitz historisch verschleiert haben. Anstatt diese Gewalt anzuerkennen, wird diese in der politischen Ökonomie mythologisiert und naturalisiert. Für Marx hat das Konzept der ursprünglichen Akkumulation eine materiale Geschichte. Es ist die vorkapitalistische Bedingung für kapitalistische Produktion. Insbesondere identifiziert Marx diesen Prozess mit der Vertreibung von Landleuten und indigenen Völkern von deren Land, welche auf physischen wie rechtlichen Grenzziehungen, auf kolonialer Enteignung oder auf Maßnahmen wie z. B. Gesetzen gegen das Vagabundieren im England des 16. Jahrhunderts basiert. Marx' These besteht darin, dass die Vorbedingung für die gesellschaftliche Ausweitung des Kapitalismus gerade in der vorherigen Vertreibung dieser Gruppen von ihrem Land sowie im Verlust ihres gewohnheitsrechtlichen Rechtsstatus zu sehen ist: Keine Ausdehnung von Privatbesitz und damit auch von Kapitalismus ohne diese Formen des Ausschlusses von Menschen.

Meine eigene These ist es nun, dass wir Grenzen als bewegliche Instrumente begreifen sollten, die nicht nur zu Zwecken wirtschaftlicher Akkumulation, sondern, noch weiter gefasst, zu Zwecken sozialer Akkumulation eingesetzt werden - ich nenne dies „Ausdehnung durch Ausschließung“ (expansion by expulsion). Die Idee der Ausdehnung durch Ausschließung entwickelt das Konzept der ursprünglichen Akkumulation fort: So stellt der Prozess der Enteignung von Personen und Gruppen, die ihres sozialen Status beraubt werden (Ausschließung), um eine bestimmte Form sozialer Bewegung voranzutreiben (Ausdehnung), keineswegs ein Alleinstellungsmerkmal des kapitalistischen Regimes sozialer Bewegung dar. Dieser Prozess lässt sich bereits in frühen Gesellschaften beobachten, deren zunehmende Bestellung von Land (territoriale Ausdehnung), zumal in der Kombination mit Technologien der Einfriedung und Abzäunung, ebenfalls Teile der Bevölkerung vertrieben und ausgeschlossen (territoriale Enteignung) hat. Dies 
trifft zum Beispiel auf Jäger und Sammler zu, deren Gebiete landwirtschaftlich erschlossen wurden, oder auf überzählige frühe Ackerbauern und Viehzüchter, für die ab einem gewissen Zeitpunkt kein nutzbares Land mehr übrig war. Somit ist soziale Ausschließung Bedingung für soziale Ausdehnung, und dies auf zwei Weisen: Sie ist einmal eine interne Bedingung, die das Entfernen von Bevölkerungsteilen gestattet, sobald gewisse Höchstgrenzen, so etwa hinsichtlich der Anbaukapazität eines bestimmten Gebietes, erreicht sind; derartige Ausschließung ist daneben auch eine externe Bedingung, die das Entfernen von Bevölkerungsteilen ermöglicht, die ursprünglich außerhalb dieser Gebiete angesiedelt sind - und zwar in dem Moment, in dem bestimmte Gruppen in der Lage sind, sich nach außen, d.h. auf die Böden anderer Gruppen, auszudehnen. In dem hier skizzierten Fall wurde territoriale Ausdehnung somit dadurch möglich, dass ein Teil der Bevölkerung als Nomad*innen ausgeschlossen und in umliegende Berg- und Wüstenregionen vertrieben wurde.

Später sehen wir dieselbe Logik in der antiken Welt am Werk, deren maßgebliche politische Form, der Staat, nicht ohne die materielle Technologie der Grenzmauer möglich geworden wäre. Mauern dienten sowohl dazu, feindliche Gruppen abzuwehren, als auch dazu, versklavte Menschen (also die erhebliche Menge von „Barbaren“, politisch Enteigneten aus dem gesamten Mittelmeerraum und dem Mittleren Osten) gefangen zu halten. Die sozialen Bedingungen für die Ausdehnung einer politischen Ordnung, darunter Kriegsführung, Kolonialismus und weitreichende öffentliche Bauvorhaben, bestanden auch hier in Ausschließungen von Bevölkerungsgruppen, die durch politische (Grenz-)Macht ein- bzw. ausgemauert wurden. Dass derartige Techniken im Verlauf der Geschichte wie auch heute auftreten, habe ich an anderer Stelle zu zeigen versucht (siehe Nail 2016).

Ein weiterer Unterschied zwischen früheren Theorien ursprünglicher Akkumulation und der hier vorgestellten, umfassenderen Neuformulierung besteht darin, dass die von Marx geschilderten Prozesse von Vertreibung und Beraubung nicht allein in territorialer oder rechtlicher Gestalt auftreten und dass die damit verbundene Ausdehnung nicht ausschließlich ökonomischer Natur ist. Denn Ausschließung bedeutet nicht länger einfach, dass Menschen von ihrem Land vertrieben werden, wenngleich dies immer noch häufig der Fall ist. Es bedeutet vielmehr auch, dass Menschen ihrer politischen Rechte beraubt werden, indem etwa Städte abgeschottet werden, bestimmte Gruppen durch Techniken der Zelle, d.h. der Einkerkerung kriminalisiert oder durch Identifizierungstechniken und checkpoints in ihren Zugangsmög- 
lichkeiten zum Arbeitsmarkt beschnitten werden. Ausschließung erweist sich dabei als der jeweilige Grad, zu dem einem politischen Subjekt ein bestimmter (Mitglieds-)Status innerhalb einer sozialen Ordnung genommen bzw. vorenthalten wird. Entsprechend erweitern solche Ordnungen ihre Machtbereiche auch auf mehrere maßgebliche Arten: Sie tun dies durch Akkumulation von Territorien sowie durch Ausdehnung politisch-rechtlicher und gewinnorientierter wirtschaftlicher Maßnahmen. Worin sich die Theorie ursprünglicher Akkumulation und diejenige der Ausdehnung durch Ausschließung ähnlich sind, ist die Feststellung, dass umfangreiche Ausdehnungen soziokinetischer Macht auf vorhergehende (oder „ursprüngliche“) Gewaltmaßnahmen kinetisch-sozialer Ausschließungen angewiesen sind. In diesem Zusammenhang stellt die Grenze diejenige materielle Technologie bzw. dasjenige soziale Regime dar, das Ausschließungen dieser Art direkt umsetzt. Das Konzept der ursprünglichen Akkumulation erweist sich somit als angemessener Zugang zu lediglich einer bestimmten historischen Konfiguration einer allgemeineren Grenzlogik, die in der Entstehung und Erneuerung früherer Gesellschaften am Werk ist.

Kurzum, zu den materialen kinetischen Voraussetzungen zur Ausdehnung von Gesellschaften gehört der Einsatz von Grenzen (Zäunen, Mauern, Zellen und checkpoints), welche systematisch ein Geflecht territorial, politisch, rechtlich und wirtschaftlich marginalisierter Minderheiten produzieren, die sich möglichst reibungslos und je nach Bedarf verschieben und in Zirkulation versetzen lassen. Ebenso wie die Minderheit der Vagabundierenden durch Ein-/Ausschließungen enteignet und in ein wirtschaftliches Proletariat verwandelt wurde, so verfügt auch jedes andere dominierende gesellschaftliche System über seine jeweils eigene Struktur der Ausdehnung durch Ausschließung.

\section{Ein aktuelles Beispiel: Klimawandel als Waffe ursprünglicher Akkumulation}

Bekanntermaßen wirkt sich der Klimawandel unverhältnismäßig negativ auf ärmere Länder und auf people of color aus, während er unverhältnismäßig positive Effekte für Länder hat, die von der prekären Situation von Klimamigrant*innen, einer eminent ausbeutbaren „Reservearmee“ von Arbeitskräften, profitieren. Diese Asymmetrie ist das Ergebnis der langen Geschichte eines kapitalistischen Kolonialismus und Rassismus, die sich heute im Migrationsmanagement mithilfe von Grenzen fortsetzt. Gegenwärtige glo- 
bale Migrationsbewegungen lassen sich daher nicht allein unter Verweis auf natürliche klimatische Ursachen erklären (siehe Hulme 2011): Die Figur des „Klimaflüchtlings“ ergreift nicht einfach die Flucht vor veränderten klimatischen Bedingungen (zu verschiedenen historischen und gegenwärtigen Figuren von Migrant*innen siehe Nail 2015), findet diese Flucht doch vielmehr unter postkolonialen Bedingungen statt, d.h. unter Bedingungen von geopolitischer Gewalt und von Rassismus. Die Bezeichnung „Klimaflüchtling“ selbst dient dazu, diese tatsächlichen kinopolitischen Bedingungen sozialer Zirkulation zu verschleiern, die solche Gruppen überhaupt erst verletzlich machen und der Gefahr der Vertreibung aussetzen.

Klimawandel wird insofern zu einer Waffe ursprünglicher Akkumulation bzw. einer Waffe der „Ausdehnung durch Ausschließung“, als der Westen durch diesen bedingt einen Machtzuwachs erzielt: Menschen sind zur Aufgabe ihrer bisherigen Bewegungsmuster gezwungen und geraten so unter den Zugriff von spezifischen, von diesem festgelegten Bedingungen sozialer Reproduktion. Ausschließung nimmt dabei vierfach Gestalt an: Als territoriale Ausschließung führt sie für Migrant*innen zum Verlust von Heimat und Besitz; als politische Ausschließung zum Verlust von bürgerlichen Partizipationsrechten; als juridische Ausschließung zum Verlust ihres Anspruchs auf rechtlichen Status; sowie als wirtschaftliche Ausschließung zum Verlust ihres Rechts auf diejenigen Mittel, die für Auskommen und Produktion erforderlich sind. Umgesetzt wird diese vierfache Ausschließung durch Grenzen, welche die notwendige Bedingung dafür sind, dass es zu einer unmittelbaren Aneignung von verletzlichen, billigen migrantischen Körpern und somit zu einer derartigen Ausdehnung sozialer Macht kommen kann.

Nationalismus, Fremdenhass und Rassismus spielen im Zusammenhang mit dem Grenzziehungsprozess ursprünglicher Akkumulation auf struktureller Ebene eine Rolle, weil sie die Arbeit und das Leben von Arbeitsmigrant*innen gesellschaftlich ab- bzw. entwerten. Denn würden Migrant*innen ankommen, ohne durchweg rassifiziert und anderweitig diskriminiert zu werden, wäre deren Arbeitskraft zu wertvoll, um kapitalistische Investitionen überhaupt erst zu deren Vereinnahmung und Inbesitznahme zu veranlassen. Der Kapitalismus bringt Klimawandel somit auf drei Arten unter Vorzeichen eines Grenzkolonialismus in Stellung: Erstens liegen die historischen Ursprünge gegenwärtigen Klimawandels wesentlich selbst im Kolonialismus (etwa im Zusammenhang mit Ölförderung oder mit durch Sklaverei gesteigerter industrieller Produktion); zweitens sind historisch kolonialisierte und indigene Völker wegen des Klimawandels überpropor- 
tional zur Migration gezwungen; und drittens werden ebendiese Gruppen beispielsweise als gefährliche, barbarische boat people rassifiziert (siehe Giuliani 2017; Goldberg 2017; Moore 2015).

Ähnlich wie die ursprüngliche Akkumulation läuft Klimawandel so verstanden nicht nur auf die Ent- bzw. Aneignung von Menschen und von günstiger Arbeitskraft hinaus, geht es doch dabei auch um die direkte Aneignung von billigem Land; beide Aspekte gehen Hand in Hand. So werden zur gleichen Zeit, zu der Klimawandel Menschen vertreibt, Landflächen, Gewässer und Waldgebiete für die privatisierte Rohstoffindustrie frei, die zuvor in Besitz der Vertriebenen standen. In dem Maße, wie sich das Klima verändert, lassen sich Gebiete, zu welchen zuvor der Zugang fehlte, für expandierende, im Überfluss mit billiger Arbeitskraft versorgte neue Märkte erschließen. Dies beinhaltet auch Märkte für die Sicherheitsindustrie, so in Form von neuen Grenztechnologien, von Zäunen oder Drohnen und einer Menge mehr (man denke etwa nur an die Privatisierung und Gentrifizierung von New Orleans in der Folge von Hurrikan Katrina). Anders gesagt ist es durchaus vorstellbar, dass Klimawandel gerade nicht das Ende des Kapitalismus bedeutet, sondern mit dessen zweiter Luft oder mit dessen Wiedergeburt durch den Einsatz von Grenzen zusammenfällt.

Warum also sollten wir annehmen, dass der Klimawandel notwendigerweise auf ein Ende des Kapitalismus hinausläuft (siehe z. B. Klein 2007), zumal dieser doch gerade Desaster liebt? Denn wenn sich alles kommodifizieren lässt, gibt es für diesen keine absoluten natürlichen Grenzen, sondern lediglich relative Grenzen des zu erzielenden Profits. Heute befinden wir uns zweifellos an der Schwelle zu einer derartigen Höchstgrenze, was Jason Moore der „tendency of the ecological surplus to fall“ (Moore 2015, 13) zuschreibt. Alles, das bzw. jede*r, die/der sich ohne größere Schwierigkeiten aneignen ließ, wurde zu Zeiten des Kolonialismus auch tatsächlich einverleibt - ob nun Öl, Altholzbestände oder versklavte Menschen. Dagegen setzen sich Menschen heute mit Forderungen nach mehr Geld und mehr Rechten zur Wehr; und die Mineralien zu fördern, die noch übrig sind, ist zu teuer geworden. Das ist der Grund, warum sich kapitalistische Bestrebungen gegenwärtig vermehrt auf Finanzspekulationen zurückgezogen haben. Wenn es nur einen Weg gäbe, so träumt der Kapitalismus, in irgendeiner Weise große Mengen von Menschen kostengünstig von ihrem Land zu entfernen, ihre Arbeit durch Grenzziehungen zu entwerten und sich diese anschließend zu eigen zu machen - oder in anderen Worten: Wenn Klimawandel nicht ohnehin existierte, so wäre es für den Kapitalismus unerlässlich, diesen zu 
schaffen. Nur gut für den Kapitalismus, dass Klimawandel tatsächlich existiert, da er diesen ja tatsächlich geschaffen hat. Und so bilden Migrant*innen heutzutage „eine disponible industrielle Reservearmee, die dem Kapital ganz so absolut gehört, als ob es sie auf seine eigenen Kosten großgezüchtet hätte“ (Marx 1968, 661).

\section{Schluss}

Wir leben in einer Welt von Grenzen. Territoriale, politische, rechtliche und ökonomische Grenzen aller Art definieren, und dies geradezu im Wortsinn, sämtliche Aspekte gesellschaftlichen Lebens im 21. Jahrhundert. Den Hymnen auf die Globalisierung und der zunehmenden Notwendigkeit globaler Mobilität zum Trotz, existieren nun mehr Arten von Grenzen als je zuvor in der Geschichte. Wenn wir den höchst beweglichen Geschehnissen unserer Zeit gerecht werden wollen, müssen wir anfangen, ernsthafter über die Bewegungen von Grenzen selbst, d.h. über diese als aktive Faktoren in Grenzziehungsprozessen, nachzudenken. Wir müssen anfangen, über die Kinopolitik der Grenzen nachzudenken.

\section{Literatur}

Agamben, Giorgio. 1998. Homo Sacer: Sovereign Power and Bare Life, Übersetzung von Daniel Heller-Roazen. Stanford: Stanford University Press.

Ancel, Jacques. 1936. „Les frontières, étude de géographie politique“. Collected Courses of the Hague Academy of International Law, volume 55. Leiden und Boston: Brill-Nijhoff. http://dx.doi.org/10.1163/1875-8096 pplrdc ej.9789028609228.203 297.

Bauman, Zygmunt. 2013. Liquid Modernity. Hoboken: Wiley.

Brown, Wendy. 2010. Walled States, Waning Sovereignty. New York: Zone Books.

Castells, Manuel. 1996. The Rise of the Network Society. Malden: Blackwell.

Cole, Phillip. 200o. Philosophies of Exclusion: Liberal Political Theory and Immigration. Edinburgh: Edinburgh University Press.

Giuliani, Gaia. 2017. „Afterword: Life Adrift in a Postcolonial World“. In Life Adrift: Climate Change, Migration, Critique, herausgegeben von Andrew Baldwin und Giovanni Bettini, 227-242. New York: Rowman \& Littlefield International.

Goldberg, Daniel Theo. 2017. „Parting Waters: Seas of Movement“. In Life Adrift: Climate Change, Migration, Critique, herausgegeben von Andrew Baldwin und Giovanni Bettini, 99-114. New York: Rowman \& Littlefield International. 
Hinnant, Lori, und Bram Janssen. 2018. „56,800 Migrant Dead and Missing: ,They Are Human Beings““, Associated Press, 1. November 2018. www.apnews.com/ e 509e15£8bo74b1d984f97502eab6a25.

Hulme, Mike. 2011. „Reducing the Future to Climate: A Story of Climate Determinism and Reductionism“. Osiris 26 (1): 245-266.

International Organization for Migration (IOM). 2009. Compendium of IOM's Activities in Migration, Climate Change, and the Environment. www.iom.int/jahia/ webdav/shared/shared/mainsite/activities/env degradationIcompendium climate change.pdf. Letzter Zugriff: 24.11.2018.

International Organization for Migration (IOM). 2010. World Migration Report 2010: The Future of Migration: Building Capacities for Change. https://publications.iom:int/system/files/pdf/wmr 2010 english.pdf. Letzter Zugriff: 24.11. 2018.

Johnson, Corey, et al. 2011. „Interventions on Rethinking ,the Border in Border Studies“. Political Geography 30 (2): 61-69.

Klein, Naomi. 2007. The Shock Doctrine: The Rise of Disaster Capitalism. New York: Metropolitan Books/Henry Holt.

Latour, Bruno. 2018. Down to Earth: Politics in the New Climatic Regime. Oxford: Polity Press.

Marx, Karl. 1976 [1867]. Capital: A Critique of Political Economy. Übersetzung von Ben Fowkes. New York: Penguin Books.

Marx, Karl. 1968 [1867]. Das Kapital. Berlin: Dietz.

Moore, Jason. 2015. Capitalism in the Web of Life: Ecology and the Accumulation of Capital. London: Verso.

Nail, Thomas. 2015. The Figure of the Migrant. Stanford: Stanford University Press.

Nail, Thomas. 2016. Theory of the Border. Oxford: Oxford University Press. Newman, David. 2003. „On Borders and Power: A Theoretical Framework“. Journal of Borderlands Studies 18 (1): 13-25.

Prescott, J.R.V. 1987. Political Frontiers and Boundaries. London: Allen \& Unwin.

Smith, Adam. 2003 [1776]. The Wealth of Nations. New York: Bantam Classic.

United Nations Development Program (UNDP). 2009. Human Development Report 2009: Overcoming Barriers: Human Mobility and Development. http://hdr. undp.org/sites/default/files/reports/269/hdr 2009 en complete.pdf. Letzter Zugriff: 24.11.2018.

Urry, John. 2000. Sociology Beyond Societies: Mobilities for the Twenty-First Century. London: Routledge.

Vaughan-Williams, Nick. 2009. Border Politics: The Limits of Sovereign Power. Edinburgh: Edinburgh University Press. World Health Organization (WHO). 2015. Migrant Health. www.who.int/migrants/en/. Letzter Zugriff: 24.11.2018. 